\title{
Herausforderungen in der psychosozialen Versorgung am Beispiel der psychotherapeutischen Universitätsambulanz der Sigmund Freud PrivatUniversität - Eine Klientelanalyse
}

\author{
Manfred Reisinger · Florian Knasmüller · Jan Aden · Brigitte Sindelar
}

Angenommen: 30. April 2021 / Online publiziert: 11. Juni 2021

(C) Der/die Autor(en) 2021

Zusammenfassung Das Versorgungsmodell der Universitätsambulanz der SFU folgt der Zielsetzung, einen niederschwelligen Zugang zur Psychotherapie zu eröffnen, indem niedrige Tarife und kurze Wartezeiten für die Patient*innen die Regel darstellen. Um die Klientelstruktur der Ambulanz systematisch beschreiben zu können, wurden über einen Erhebungszeitraum von 2015-2018 Patient*innenmerkmale einer Untersuchungsstichprobe von $n=629$ Patient*innen erhoben. Auf Basis soziodemographischer und krankengeschichtlicher Informationen sowie der aktuellen Lebensqualität (WHOQOL) wurde eine Clusteranalyse nach der WARD-Methode durchgeführt: Es konnten vier inhaltlich plausible, empirisch trennscharfe Klient*innen-Cluster eruiert werden, die sich hinsichtlich der Dimensionen Bildungsabschluss, Hauptdiagnose, Erwerbstätigkeit und Migrationshintergrund signifikant unterscheiden. Die Cluster definieren sich hinsichtlich der Diagnosen in drei Cluster mit den Hauptdiagnosen F3 (Affektive Störungen), F4 (Neurotische, Belastungs- und somatoforme Störungen), F6 (Persönlichkeitsstörungen) und einen Cluster mit Patient*innen mit der Diagnose F3 oder F4. Dieser $(n=178)$ hebt sich als einer von Indikatoren hoher Vulnerabilität geprägter von den anderen drei Clustern ab. Die Patient*innen dieses Clusters sind mittleren Alters, überwiegend erwerbslos, weisen ein niedriges Bildungsniveau auf und leben mit betreuungspflichtigen Kindern in einem Haushalt und berichten eine niedrige Lebensqualität.

Bemerkenswert ist die Altersstruktur in dieser Stichprobe mit über $72 \%$ von Patient*innen in der Lebensphase der Emerging Adulthood in den anderen drei Clustern. Diskutiert werden die Implikationen der Er-

M. Reisinger $(\bowtie) \cdot$ F. Knasmüller · J. Aden · B. Sindelar Sigmund Freud PrivatUniversität, Wien, Österreich manfred.reisinger@sfu.ac.at gebnisse für die psychotherapeutischen Ausbildungscurricula sowie für die Relevanz von Universitätsambulanzen für die psychotherapeutische Versorgung einer Klientel, die in dieser Lebenssituation wohl keine Möglichkeit zur Inanspruchnahme einer großteils selbstfinanzierten Psychotherapie fände.

Schlüsselwörter Psychosoziale Versorgung • Psychotherapie - Universitätsambulanz . Klientelanalyse $\cdot$ Versorgungsforschung

Challenges in psychosocial support by example of the psychotherapeutic outpatient clinic of Sigmund Freud University - a clientele analysis

Summary The care model of the University Outpatient Clinic of the SFU follows the objective of providing a low-threshold access to psychotherapy at low fees and short waiting times. In order to systematically describe the clientele structure of the outpatient clinic, patient characteristics of a study sample of $n=629$ patients were collected over a survey period of 2015-2018. Based on sociodemographic and medical history information as well as current quality of life (WHOQOL), a cluster analysis was performed using the WARD method: Four plausible, empirically distinct clusters of clients were identified, which differ significantly with regard to the dimensions of educational level, main diagnosis, employment and migration background. The clusters are defined in terms of diagnoses into three clusters with the main diagnoses F3 (affective disorders), F4 (neurotic, stress and somatoform disorders), F6 (personality disorders) and one cluster with patients with the diagnosis F3 or F4. This cluster ( $n=178)$ stands out from the other three clusters as characterized by indicators of high vulnerability. The patients in this cluster are middle-aged, predominantly unemployed, have a low level of ed- 
ucation, live in a household with children and report a low quality of life. Of note is the age structure in this sample with over $72 \%$ of patients* in the Emerging Adulthood life stage in the other three clusters. The implications for psychotherapeutic training as well as for university outpatient clinics for the psychotherapeutic care of a clientele who would probably not find the opportunity to use a self-financed psychotherapy are outlined.

Keywords Psychosoziale Versorgung • Psychotherapie · Universitätsambulanz · Klientelanalyse · Versorgungsforschung

\section{Psychotherapie: Situation und Bedarf in Österreich}

Psychische Erkrankungen stellen für das österreichische Gesundheitssystem eine wachsende Herausforderung dar. Etwa 1,2 Mio. Österreicher*innen sind laut einer Presseaussendung des Berufsverbands Österreichischer Psychologen im Jahr 2019 von einer solchen Erkrankung betroffen.

Die damit einhergehenden Folgen ziehen einen beachtlichen volkwirtschaftlichen Schaden nach sich: Beispielhaft seien hier die Krankenstände sowie die Frühpensionierungen, die in Folge psychischer Erkrankungen auftreten, kurz genannt: Im Jahr 2018 betrug die durchschnittliche Dauer eines Krankenstandes aufgrund der übergeordneten Diagnose „Psychische und Verhaltensstörungen“ 34,6 Tage und lag damit weit über dem Mittelwert von 9,6 Tagen, der insgesamt über alle Diagnosen errechnet wurde (Statistik Austria 2018). Die drastische Zunahme an psychischen Erkrankungen zwischen den Jahren 2008 und 2018 zeigt nicht zuletzt ein Vergleich der Zahlen der Krankenstandsfälle aufgrund psychischer Störungen: Waren es 2008 noch 22,2 Fälle, bezogen auf 1000 Erwerbstätige, so stieg die Zahl 2018 bereits auf 33,5 (Hauptverband der österreichischen Sozialversicherungsträger 2019). Besonders kostenintensiv gestalten sich die Auswirkungen im Bereich der Frühpensionierungen: So zeigte etwa der Endbericht einer im Jahr 2016 präsentierten Studie über Invaliditätspensionen (I-Pensionen) in Österreich, dass bei Männern im Jahr 2014 bereits ein Viertel (26\%), bei Frauen knapp die Hälfte $(46 \%)$ aller unbefristeten I-Pensionen auf psychischen Erkrankungen gründeten. Noch drastischer stellte sich die Situation bei den befristeten Invaliditätspensionen dar: Bei den Männern betrug der Anteil hier $57 \%$, bei den Frauen waren es sogar $69 \%$ (Czypionka et al. 2016).

Der volkswirtschaftliche Gesamtschaden, den psychische Erkrankungen jährlich in Österreich verursachen, beträgt laut dem Berufsverband Österreichischer PsychologInnen (BÖP 2019) aktuell zwölf Milliarden Euro.

\section{Psychotherapeutische Versorgung}

Valide Daten in Bezug auf die tatsächliche Situation psychotherapeutischer Versorgung in Österreich sind schwer zu generieren. Das liegt sowohl an den unterschiedlichen Versorgungsmodellen als auch an der Tatsache, dass die österreichische Gesundheitskasse bis dato keinen Gesamtvertrag für Psychotherapie mit dem Berufsstand aushandeln konnte und damit die Daten privat finanzierter Psychotherapien in den Statistiken fehlen. Eine der letzten großen Erhebungen zur psychotherapeutischen Versorgung seitens des Hauptverbandes der Sozialversicherungsträger (2011) wurde im Jahr 2009 durchgeführt: 900.000 Menschen nahmen in diesem Jahr Leistungen der Krankenkassen wegen psychischer Leiden in Anspruch, 840.000 Patient*innen erhielten verschreibungspflichtige Psychopharmaka. Dabei gaben die Krankenkassen für psychoaktive Medikamente 250 Mio.€ aus. 35.000 Menschen wurden die Kosten für Psychotherapie im Rahmen der Sachleistungsversorgung der Krankenkassen erstattet, weitere 30.000 erhielten den - damals festgelegten - Kostenzuschuss von 21,80€ pro Psychotherapiesitzung. Insgesamt wendeten die Kassen damals für Psychotherapie und psychotherapeutische Medizin 63 Mio. $€$ auf - somit lediglich ein Viertel der Kosten, die für Psychopharmaka ausgegeben wurden. Die Zahl erscheint vergleichsweise niedrig, bedenkt man, dass die Wirksamkeit von Psychotherapie im Zuge der vergleichenden Psychotherapiewissenschaft bereits Jahrzehnte vor dem Berichtsjahr 2009 verifiziert werden konnte (vgl. z. B.: Luborsky et al. 1975; Smith und Glass 1977; Glass et al. 1980; Shapiro und Shapiro 1982). Aktuellere Beiträge zur Wirksamkeitsforschung von Psychotherapie finden sich u. a. in den Standardwerken von Gelo und Manzo (2015) und Lambert (2013). Der fortlaufende Trend zur Psychopharmakotherapie bildet sich ebenfalls in einem Bericht des Bundesministeriums für Gesundheit über das österreichische Gesundheitswesen im internationalen Vergleich ab: So gehörten im Jahr 2012 Antidepressiva in Österreich zur Gruppe der zehn umsatzstärksten Wirkstoffgruppen (Habimana et al. 2015, S. 26).

In Österreich stehen Menschen mit psychischen Leiden unterschiedliche Versorgunsmodelle zur Verfügung (Riedel 2015). Neben stationären Einrichtungen, sozialpsychiatrischen Zentren, Tageskliniken mit psychotherapeutischem Angebot und niedergelassenen Psychotherapeut*innen stellen vor allem eine Vielzahl an zum Teil spezialisierten Ambulatorien eine bedeutende Säule in der psychotherapeutischen Versorgungslandschaft dar. Das Angebot umfasst dabei sowohl Ambulanzen der österreichischen Gesundheitskassa als auch Einrichtungen von öffentlichen und privaten Kliniken, Vereinen und psychotherapeutischen Ausbildungsinsitutionen. Individuelle Einzelverträge mit dem Sozialversicherungsträger regeln dabei klar definierte Stundenkontingente, die von der 
Krankenkasse entweder komplett oder zum Teil finanziert werden. Eine Sonderstellung nehmen dabei die Universitätsambulanzen ein, die im Unterschied zu den vorher genannten Einrichtungen neben ihrem psychotherapeutischen Versorgungsauftrag auch die Verpflichtung zu Forschung, Ausbildung und Evaluation tragen.

Nicht selten entscheidet in Österreich das individuelle Einkommen von Patient*innen, ob eine Psychotherapie überhaupt in Anspruch genommen werden kann. Die österreichische Sozialversicherung verwies bereits 2012 (Klein, et al. 2012, S. 18) auf die Tatsache, „[...] dass der Bedarf an Psychotherapie höher ist als von den Krankenversicherungsträgern bisher finanziert werden konnte“. Aktuell beträgt der Kostenzuschuss, den die österreichische Gesundheitskassa bei privat finanzierter Psychotherapie gewährt, 28,00€. Diese Unterstützung wird allerdings nur nach einem Antragsverfahren genehmigt, das zum Teil höchstpersönliche Informationen über die*den Antragssteller*in verlangt. Bei durchschnittlichen Behandlungskosten von 80 bis $120 €$ pro psychotherapeutische Sitzung kann diese Versorgungsform im niedergelassenen Bereich gerade für den einkommensschwachen Bevölkerungsteil nicht als niederschwellig bezeichnet werden.

Auch der Abschlussbericht einer repräsentiven Stichprobenerhebung zur Prävalenz psychischer Störungen und der Versorgung psychisch Kranker in Österreich (Wancata 2017, S. 165) stellte fest, „[...] dass 57,5\% der psychisch Kranken keinerlei Behandlung für ihre Krankheit erhielt“. Das heißt, dass über die Hälfte aller psychisch kranken Menschen in Österreich weder eine psychotherapeutische noch eine fachärztlich psychiatrische Behandlung in Anspruch nehmen konnte. Diese Daten belegen einerseits den hohen Bedarf an psychotherapeutischen Behandlungsplätzen, andererseits zeigt sich die Notwendigkeit niederschwellig verfügbarer Versorungunsgmodelle.

\section{Die psychotherapeutische Universitätsambulanz der Sigmund Freud Privatuniversität Wien}

Eine vergleichende Umfrage über Kernmerkmale psychotherapeutischer Hochschulambulanzen an 53 Standorten in Deutschland kam zu dem Ergebnis, dass jährlich über 10.000 neue Patient*innen aus dem Kinder-, Jugendlichen- und Erwachsenenbereich in diesen Institutionen behandelt werden (Hoyer et al. 2015). Aus diesen Zahlen kann geschlussfolgert werden, dass psychotherapeutische Universitätsambulanzen eine wichtige Säule für die notwendige psychotherapeutische Versorgung darstellen.

Die Sigmund Freud Privatuniversität (SFU) betreibt seit ihrer Akkreditierung im Jahr 2005 eine psychotherapeutische Universitätsambulanz. Mit dem Spannungsfeld zwischen hohem Bedarf und Knappheit an psychotherapeutischen Behandlungsplätzen einerseits und dem niederschwelligen Versorgungsangebot der Universitätsambulanz der SFU andererseits gehen spezifische Herausforderungen einher. Diese liegen vordergründig im Bereich der Klientelstruktur, die sowohl hinsichtlich der Lebensqualität als auch der sozialen Lage von starker Belastung geprägt ist. Die vorliegende Untersuchung zielt darauf $a b$, die spezifischen Belastungserscheinungen der Klientel der psychotherapeutischen Ambulanz abzubilden, um in weiterer Folge konkrete Implikationen für die Versorgungspraxis und auch die Ausbildungspraxis ableiten zu können.

\section{Forschungsfragen}

Im Rahmen von Evaluation, Forschung und Qualitätsmanagement muss zur Gewährleistung eines an den Bedarf angepassten psychotherapeutischen Angebots auch der Frage nachgegangen werden, welche Gruppen von Patient*innen das Angebot für sich nützen können - bzw. welche nicht. Die offen gerichtete Forschungsfrage für die vorliegende Untersuchung lautet daher:

Durch welche psychosozialen Charakteristika ist die Klientel der psychotherapeutischen Universitätsambulanz der Sigmund Freud PrivatUniversität gekennzeichnet?

\section{Material und Methode}

Die in der vorliegenden Analyse verwendeten Daten stellen einen Auszug der obligatorischen Dokumentation der psychotherapeutischen Universitätsambulanz der Sigmund Freud PrivatUniversität dar und umfassen soziodemographische Informationen sowie ausgewählte Indikatoren psychosozialer Gesundheit bei Erstaufnahme der Patient*innen (Zeitraum 2015-2018).

\section{Erhebungsinstrumente}

Aus dem Set der erhobenen Patient*innendaten wurden in der vorliegenden Untersuchung die Variablen Geschlecht, Stand der Erwerbstätigkeit, Familienstand, Bildungsstand, Migrationshintergrund, Anzahl der Kinder im gemeinsamen Haushalt sowie das Alter bei Anmeldung einbezogen.

Neben den soziodemographischen Informationen wurden Indikatoren der aktuellen Lebensqualität der Patient*innen erhoben. In der gegenständlichen Auswertung wird dabei auf den an der Ambulanz routinemäßig eingesetzten WHOQOL-BREF (Angermeyer, Kilian \& Matschinger 2020) mit einer Skalierung von 0-100 zurückgegriffen.

Die Information bezüglich der Hauptdiagnose der Patient*innen entstammt einem differentialdiagnostischen Prozess durch die behandelnden Psychotherapeut*innen und folgt dem Klassifikationsschema des ICD-10. 


\section{Datenanalyse}

Zur Identifikation einer Patient*innen-Typologie wurde auf Basis der dichotomisierten Variablen $G e-$ schlecht (weiblich/männlich), Erwerbstätigkeit (regelmäßiges Nachgehen einer beruflichen Tätigkeit ja/ nein), Familienstand (verpartnert/nicht verpartnert), Bildungsstand (Matura ja/nein), Migrationshintergrund (ja/nein), sowie der Dummy-codierten Variable Hauptdiagnose (F3, F4, F6) und der intervallskalierten Merkmale Alter bei Anmeldung (in Jahren und damit intervallskaliert), Anzahl der Kinder im gemeinsamen Haushalt sowie dem Testscore zur Lebensqualität (WHOQOL-BREF) eine Hierarchische Clusteranalyse durchgeführt. Dabei wurde auf die WARD-Methode und die quadrierte euklidische Distanz zurückgegriffen.

Nach Identifikation der Cluster wurden diese hinsichtlich der eingespielten Merkmale inferenzstatistisch verglichen. Bei intervallskalierten Merkmalen wurden einfaktorielle Varianzanalysen (ANOVA) und für die dichotomen Variablen $\chi^{2}$-Analysen durchgeführt, um die Clusterlösung auch auf inhaltlicher Ebene definieren zu können. Um die Relevanz der zur Clusterung herangezogenen Merkmale untereinander besser vergleichbar zu machen und deren Höhe abschätzen zu können, wurden ergänzend zu den $\chi^{2}$-Analysen der jeweilige Kontingenzkoeffizient (C) sowie der korrigierte Kontingenzkoeffizient (Ckorr.) berechnet und bezogen auf die Varianzanalysen die Effektstärke $\eta^{2}$ geschätzt.

Das Signifikanzniveau wurde dabei auf $5 \%$ festgelegt. Alle Berechnungen erfolgten mit der Auswertungssoftware IBM-SPSS (Version 25).

\section{Stichprobe}

Die Untersuchungsstichprobe von insgesamt $n=629$ Personen stellt eine Teilstichprobe des gesamten Patient*innenaufkommens der psychotherapeutischen Universitätsambulanz im Zeitraum 2015-2018 dar. Als Einschlusskriterien fungiert die Vollständigkeit der Datenangaben bzgl. aller Charakterisierungsmerkmale, die der vorgenommenen Clusterung zugrunde gelegt wurden(siehe Erhebungsinstrumente und Datenanalyse). Obgleich die Teilstichprobe nicht das gesamte Patient*innenaufkommen umfasst, bildet diese die Klientel der gesamten Erwachsenenambulanz für den Erfassungszeitraum (ohne wesentliche Abweichungen) adäquat $\mathrm{ab}$.

Das durchschnittliche Alter beträgt 32,87 Jahre $(S D=11,96)$. Die Anzahl der Kinder, die mit den Patient*innen in einem gemeinsamen Haushalt leben, liegt bei $M=0,28(S D=0,68)$. Der Anteil an Personen, die entweder keine Kinder haben oder mit diesen zumindest nicht gemeinsam leben, nimmt einen Wert von $80,8 \%(n=508)$ an. Weitere soziodemographische und klinische Merkmale lassen sich aus der Tab. 1 entnehmen.
Tab. 1 Soziodemographische und klinische Merkmale der Untersuchungsstichprobe $(n=629)$

\begin{tabular}{|c|c|c|}
\hline Parameter & Anzahl (n) & Anteil (\%) \\
\hline \multicolumn{3}{|l|}{ Geschlecht } \\
\hline Weiblich & 391 & 62,2 \\
\hline Männlich & 238 & 37,8 \\
\hline \multicolumn{3}{|l|}{ Bildungsstand } \\
\hline Matura & 415 & 66 \\
\hline Keine Matura & 214 & 34 \\
\hline \multicolumn{3}{|l|}{ Erwerbstätigkeit } \\
\hline Erwerbstätig & 457 & 72,7 \\
\hline Nicht erwerbstätig & 172 & 27,3 \\
\hline \multicolumn{3}{|l|}{ Partnerschaft } \\
\hline Verpartnert & 269 & 42,8 \\
\hline Nicht verpartnert & 360 & 57,2 \\
\hline \multicolumn{3}{|c|}{ Migrationshintergrund } \\
\hline $\mathrm{Ja}$ & 153 & 24,3 \\
\hline Nein & 476 & 75,7 \\
\hline \multicolumn{3}{|l|}{ Hauptdiagnose } \\
\hline F3 & 306 & 48,6 \\
\hline F4 & 262 & 41,7 \\
\hline F6 & 61 & 9,7 \\
\hline
\end{tabular}

\section{Ergebnisse}

\section{Identifikation von Patient*innentypologien}

Auf Basis der Hierarchischen Clusteranalyse konnte eine inhaltlich plausible sowie empirisch trennscharfe vier Cluster umfassende Lösung identifiziert werden.

Dabei erweisen sich mit Ausnahme des Geschlechts und Familienstandes alle dichotomen Merkmale in den vier Clustern als signifikant different. Die deutlichsten Unterschiede zeigen sich in der Hauptdiagnose und der Erwerbstätigkeit, welche gefolgt vom Bildungsstand die mit Abstand relevantesten Variablen für die Charakterisierung der Cluster darstellen (siehe Tab. 2).

Ebenso zeigen sich bedeutsame Unterschiede in den intervallskalierten Merkmalen, welche mit jeweils $p<0,001$ hoch signifikant ausfallen. Entlang der Effektstärken lässt sich das Alter der PatientInnen als die für die Charakterisierung der Cluster relevanteste Variable identifizieren (siehe Tab. 3).

Tab. $2 \chi^{2}$-Analysen (Kontingenztafeln) der Cluster bezogen auf die kategorealen Variablen

\begin{tabular}{|l|l|l|l|l|l|}
\hline & $\mathrm{X}^{2}$ & Df & $C$ & $C$ korr & $p$ \\
\hline Geschlecht & 4,84 & 3 & 0,087 & 0,111 & 0,184 \\
\hline Erwerbstätigkeit & 280,54 & 3 & 0,555 & 0,706 & $<0,001$ \\
\hline Familienstand & 6,89 & 3 & 0,104 & 0,132 & 0,075 \\
\hline $\begin{array}{l}\text { Matura (ja/nein) } \\
\text { Hauptdiagnose }\end{array}$ & 86,99 & 3 & 0,349 & 0,444 & $<0,001$ \\
\hline $\begin{array}{l}\text { Migrationshintergrund (ja/nein) } \\
N=629\end{array}$ & 1079,32 & 6 & 0,795 & 0,945 & $<0,001$ \\
\hline$N$ & & 3 & 0,150 & 0,191 & 0,002 \\
\hline
\end{tabular}


Tab. 3 Univariaten Varianzanalysen (ANOVA) der Cluster bezogen auf die metrisch skalierten Merkmale

\begin{tabular}{|l|l|l|l|l|l|} 
& $F$ & $d f 1$ & $d f 2$ & $p$ & $\eta^{2}$ \\
\hline $\begin{array}{l}\text { Lebensqualität } \\
\text { WHOQOL_GLOBAL }\end{array}$ & 20,67 & 3 & 625 & $<0,001$ & 0,090 \\
\hline $\begin{array}{l}\text { Alter } \\
\text { (Zeitpunkt Anmeldung) }\end{array}$ & 64,80 & 3 & 625 & $<0,001$ & 0,237 \\
\hline $\begin{array}{l}\text { Anzahl Kinder } \\
\text { (gemeinsam im Haushalt) }\end{array}$ & 18,20 & 3 & 625 & $<0,001$ & 0,080 \\
\hline$N=629$ & & & & & \\
\hline
\end{tabular}

Die Clusteranalyse brachte ein Ergebnis mit vier trennscharfen und inhaltlich plausiblen Patient*innenclustern hervor, die sich signifikant hinsichtlich der Dimensionen Erwerbstätigkeit, Migrationshintergrund, Bildungsabschluss und Hauptdiagnose nach ICD-10 unterscheiden. Die in Tab. 4 abgebildeten Cluster stellen sich inhaltlich wie folgt dar: Cluster 1 $(n=201)$ beschreibt berufstätige, kinderlose Personen mit durchschnittlichem Wohlbefinden, die von neurotischen und Belastungsstörungen betroffen sind. Cluster $2(n=178)$ beinhaltet die psychosozial am stärksten belastete Gruppe von mehrheitlich erwerbslosen Personen mit niedrigem Bildungsniveau, vielen Kindern, einem niedrigen Wohlbefinden sowie neurotischen und Belastungs- oder affektiven Störungen. Cluster $3(n=61)$ bildet alleinstehende, berufstätige und kinderlose Personen ab, die bei durchschnittlichem Wohlbefinden an einer Verhaltens- oder Persönlichkeitsstörung leiden. Cluster $4(n=189)$ enthält junge, kinderlose Patient*innen mit hohem Bildungsniveau, durchschnittlichem Wohlbefinden, die von affektiven Störungen betroffen sind. Das am stärksten trennende Merkmal zwischen den Clustern stellt die Hauptdiagnose dar.

Tab. 4 macht auf den Cluster 2 aufmerksam, zu dem Personen mit einer Diagnose aus der Gruppe F3 oder F4 gehören. Dies legt die Frage nahe, was die Angehörigen dieses Cluster von Cluster 1 und Cluster 4 unterscheidet, in dem jeweils die Diagnose F4 oder F3 dominiert.

Es zeigt sich, dass höheres Alter, Arbeitslosigkeit, niedriges Bildungsniveau und der höchste Anteil an betreuungspflichtigen Kindern sowie die niedrigste Lebensqualität der Patient*innen dieser Gruppe diesen Cluster von Cluster 1 (Diagnose F4) und Cluster 4
(Diagnose F3) unterscheiden. Dies legt eine weitere Analyse nahe, wie sich die Patient*innen mit der Diagnose F4 des Cluster 2 von den Patient*innen mit derselben Diagnose im Cluster 1 unterscheiden, ebenso wie die Patient*innen mit der Diagnose F3 des Clusters 2 von denen mit derselben Diagnose des Clusters 4.

Dies wurde anhand paarweiser Gruppenvergleiche mittels $\chi^{2}$-Analysen bei dichotomen und mit t-Tests für unabhängige Stichproben bei Merkmalen metrischen Skalenniveaus überprüft.

Die interferenzstatistische Auswertung macht klar: Die Patient*innen mit der Diagnose F4 des Clusters 1 unterscheiden sich signifikant von denen mit derselben Diagnose des Clusters 2 durch die wesentlich höhere Arbeitslosigkeit und das niedrigere Bildungsniveau der Patient*innen im Cluster 2, ebenso die Patient*innen mit der Diagnose F3 des Clusters 2.

Die Lebensqualität, erfasst mit dem WHOQOLBREF, liegt bei den Patient*innen mit einer Diagnose aus der Hauptgruppe F3 des Clusters 2 in allen Skalen signifikant niedriger als bei den Patient*innen des Clusters 4 bis auf den Wert in der Skala des psychischen Wohlbefindens. Das heißt: Bei bestehender Diagnose einer affektiven Störung, in der vorliegenden Stichprobe einer depressiven Störung, trifft das niedrige Bildungsniveau und die hohe Arbeitslosigkeit mit einer Einschränkung der Lebensqualität in den Bereichen des physischen Wohlbefindens, der sozialen Beziehungen und der Lebensumwelt, nicht aber mit dem Bereich des psychischen Wohlbefindens zusammen.

Bei den Patient*innen mit einer Diagnose aus der Hauptgruppe F4 des Clusters 2 ist die Lebensqualität in allen Skalen des WHOQOL signifikant niedriger als bei den Patient*innen des Clusters 1. Das heißt: Bei bestehender Diagnose einer neurotischen, Belastungs- oder somatoformen Störung geht das niedrige Bildungsniveau und die hohe Arbeitslosigkeit mit einer Einschränkung der Lebensqualität in den Bereichen des physischen Wohlbefindens, des psychischen Wohlbefindens, der sozialen Beziehungen und der Lebensumwelt einher.

Tab. 4 Übersicht über die Differenzierung der Cluster

\begin{tabular}{|c|c|c|c|c|c|c|c|c|c|}
\hline & $\begin{array}{l}\text { Hauptdiag- } \\
\text { nose nach } \\
\text { ICD-10 }\end{array}$ & Alter & $\begin{array}{l}\text { Arbeits- } \\
\text { situation }\end{array}$ & $\begin{array}{l}\text { Bildungs- } \\
\text { niveau }\end{array}$ & WHO-QOL & Geschlecht & Partnerschaft & Kinder & $\begin{array}{l}\text { Migrationshinter- } \\
\text { grund }\end{array}$ \\
\hline Cluster 1 & F4 & $\begin{array}{l}M=30 \\
S D=9,19\end{array}$ & $\begin{array}{l}93,5 \% \\
\text { berufstätig }\end{array}$ & $\begin{array}{l}77,7 \% \\
\text { mit Matura }\end{array}$ & $\begin{array}{l}M=50 \\
S D=20,3\end{array}$ & $\begin{array}{l}67,7 \% \\
\text { weiblich }\end{array}$ & $\begin{array}{l}51,1 \% \text { nicht in } \\
\text { Partnerschaft }\end{array}$ & $\begin{array}{l}16,9 \% \text { mit Kindern } \\
\text { im Haushalt }\end{array}$ & $\begin{array}{l}27,9 \% \text { mit Migrati- } \\
\text { onshintergrund }\end{array}$ \\
\hline Cluster 2 & $\mathrm{~F} 3$ und F4 & $\begin{array}{l}M=41,9 \\
S D=14,1\end{array}$ & $\begin{array}{l}73 \% \text { arbeits- } \\
\text { los }\end{array}$ & $\begin{array}{l}60,1 \% \\
\text { ohne Matura }\end{array}$ & $\begin{array}{l}M=36,94 \\
S D=20,9\end{array}$ & & $\begin{array}{l}56,7 \% \text { nicht in } \\
\text { Partnerschaft }\end{array}$ & $\begin{array}{l}32,6 \% \text { mit Kindern } \\
\text { im Haushalt }\end{array}$ & $\begin{array}{l}19,1 \% \text { mit Migrati- } \\
\text { onshintergrund }\end{array}$ \\
\hline Cluster 3 & F6 & $\begin{array}{l}M=31,7 \\
S D=10,61\end{array}$ & $\begin{array}{l}67,2 \% \\
\text { berufstätig }\end{array}$ & $\begin{array}{l}57,4 \% \\
\text { mit Matura }\end{array}$ & $\begin{array}{l}M=47,13 \\
S D=20,6\end{array}$ & $\begin{array}{l}54,1 \% \\
\text { weiblich }\end{array}$ & $\begin{array}{l}65,6 \% \text { nicht in } \\
\text { Partnerschaft }\end{array}$ & $\begin{array}{l}16,4 \% \text { mit Kindern } \\
\text { im Haushalt }\end{array}$ & $\begin{array}{l}9,8 \% \text { mit Migrations- } \\
\text { hintergrund }\end{array}$ \\
\hline Cluster 4 & F3 & $\begin{array}{l}M=27,6 \\
S D=7,19\end{array}$ & $\begin{array}{l}95,2 \% \\
\text { berufstätig }\end{array}$ & $\begin{array}{l}81 \% \\
\text { mit Matura }\end{array}$ & $\begin{array}{l}M=51,2 \\
S D=19,3\end{array}$ & $\begin{array}{l}61,4 \% \\
\text { weiblich }\end{array}$ & $\begin{array}{l}61,9 \% \text { nicht in } \\
\text { Partnerschaft }\end{array}$ & $\begin{array}{l}10,1 \% \text { mit Kindern } \\
\text { im Haushalt }\end{array}$ & $\begin{array}{l}30,2 \% \text { mit Migrati- } \\
\text { onshintergrund }\end{array}$ \\
\hline
\end{tabular}




\section{Diskussion}

Aus den vier eruierten Clustern hebt sich Cluster 2 $(n=178)$ als jener Typ ab, der von der stärksten psychosozialen Belastung betroffen ist. Darin finden sich Personen mittleren Alters, die zu einem hohen Maß erwerbslos sind, ein niedriges Bildungsniveau aufweisen und mit betreuungspflichtigen Kindern in einem Haushalt leben. Der daraus resultierende Druck bildet sich in unterdurchschnittlichen Werten des allgemeinen Wohlbefindens ab - mit 36,94 $(S D=20,9)$ handelt es sich dabei um den niedrigsten Wert im Vergleich zu den anderen Clustern, der darüber hinaus deutlich unter dem durchschnittlichen Wert der Gesamtstichprobe $(\mathrm{MD}=47,03 ; \mathrm{SD}=21,54)$ angesiedelt ist. Personen aus besagtem Cluster suchen die Ambulanz überwiegend aufgrund neurotischer, Belastungs- oder somatoformer Störungen oder affektiver Störungen (F3 bzw. F4) auf. Daraus folgt, dass mit 28,2\% fast ein Drittel aller zur Clusteranalyse herangezogenen Patient*innen in jene Gruppe fällt, die nicht nur von besonders stark ausgeprägter psychischer, sondern auch erheblicher sozialer Vulnerabilität gekennzeichnet ist.

Auch wenn in diesem Cluster die Diagnosen aus den Gruppen F3 und F4 nicht komorbid angegeben sind, ist die Komorbidität von Angst und Depression ein vielfach berichtetes Ergebnis (vgl. zum Beispiel: Onken und Ströhle 2005; Schmidt-Traub und Lex 2005). In einer Langzeitstudie an mehr als 3000 Jugendlichen und jungen Erwachsenen finden Wittchen et al. Angststörungen und Depression in einer lebenszeitlichen Folge, in der sich die Angststörung als Risikofaktor für die Entwicklung einer komorbiden depressiven Störung darstellt: „Baseline clinical characteristics of anxiety disorders were significantly associated with an increased risk to develop major depression over the follow-up period" (Wittchen et al. 2010, S. 14). Das Zusammentreffen von depressiven Störungen und neurotischen, Belastungs- und somatoformen Störungen in Cluster 2 gibt Anlass zu psychodynamischen Überlegungen zur gegenseitigen Bedingtheit von sozialer Prekarität und psychischer Gesundheit, die offensichtlich ein Charakteristikum dieses Clusters ausmacht, einhergehend mit dem mittleren Erwachsenenalter, aber nicht mit der biographischen Gegebenheit des Migrationshintergrunds, der sich in Cluster 1 (Diagnosen aus F3) und in Cluster 4 (Diagnosen aus F4) als höher erweist. Als inhaltlich nicht relevant ist der signifikante Unterschied des Cluster 2 des höheren Anteils an betreuungspflichtigen Kindern zu sehen, da dies bei Personen im mittleren Erwachsenenalter im Unterschied zu jüngeren Personen zu erwarten ist.

Die Altersstruktur der Gesamtstichprobe macht auf einen entwicklungspsychologischen Aspekt aufmerksam: Fast $72 \%$ der Stichprobe stehen im Lebensalter des jungen Erwachsenenalters, das mittlerweile als Entwicklungsphase der „emerging adulthood“ definiert wurde und von Arnett durch fünf Charakteristika „,identity exploration, instability, self-focus, feeling inbetween and possibilities/optimism" beschrieben wird (Arnett 2015, S. ix), zugleich als „complex period with respect to mental health, because feelings of depression and anxiety are strikingly high" (ebd., S. xi). Die Altersstruktur der untersuchten Stichprobe unterstreicht dies, woraus sich die Annahme einer erhöhten psychischen Vulnerabilität in der Lebensphase der „Emerging Adulthood“ abzeichnet, was sich in Cluster 1, 3 und 4 abzeichnet.

Die Ergebnisse sind für das Versorgungmodell von psychotherapeutischen Ausbildungs- und Universitätsambulanzen in zweifacher Weise von besonderer Bedeutung: Zum Einen lassen sich aus den Befunden spezifische Implikationen für die Ausbildungskonzeption ableiten. Aus den hier vorgelegten Ergebnissen ist dies einmal die Sensibilisierung der in Ausbildung befindlichen Psychotherapeut*innen für die hohe psychische Vulnerabilität in der Lebensphase der Emerging Adulthood, in der auch der Großteil der Ausbildungskandidat*innen stehen. Damit ist zwar eine altersmäßige Passung zwischen Patient*in und Therapeut*in als häufig zu erwarten, gleichzeitig aber eine besondere Aufmerksamkeit auf die Reflexion von Übertragungs-, Gegenübertragungs- und Identifikationsprozessen erforderlich. Zum Zweiten ist dies das Wissen um das psychopathologische Naheverhältnis von Angststörungen und Depression. Und zum Dritten ist es die Kenntnis und die Beachtung der Einflussnahme von Arbeitslosigkeit, für die ein niedriges Bildungsniveau einen hohen Risikofaktor darstellt, auf die Veränderung der Persönlichkeit. So fanden beispielsweise Boyce et al. an einer großen Stichprobe von 6769 deutschen Erwachsenen im Zusammenhang mit dem Zustand der Arbeitslosigkeit bei abhängigen Stichproben eine Verringerung der Persönlichkeitsfaktoren (nach dem Big-Five-Persönlichkeitsmodell) der Offenheit für Erfahrungen und damit der Aufgeschlossenheit, der Gewissenhaftigkeit und der Verträglichkeit, zu der auch die Kooperationsbereitschaft und Empathie zu zählen sind (Boyce et al. 2015). Die Bedeutung dieser Ergebnisse nicht nur für die Lebensgestaltung dieser Patient*innen, sondern auch für die psychotherapeutische Beziehung und den Prozess ist evident.

Zum anderen sehen wir in der sozialstrukturellen Zusammensetzung der Klientel die wichtige Funktion des niederschwellig verfügbaren psychotherapeutischen Angebots der SFU-Universitätsambulanz als Teil der psychosozialen Versorgungslandschaft bestätigt, da sowohl die Lebensphase der Emerging Adulthood als auch die Situation der Arbeitslosigkeit annehmen lassen, dass in Österreich diese Patient*innen keinen Zugang $\mathrm{zu}$ einer - zumindest $\mathrm{zu}$ einem hohen Anteil - selbstfinanzierten psychotherapeutischen Behandlung hätten (Implikationen für die Notwendigkeit eines flächendeckenden kassenfinanzierten psychotherapeutischen Angebots in Österreich liegen auf der Hand, sind aber nicht Themen- 
stellung dieser Studie). Angesichts sich gegenwärtig verschärfender sozialer und gesundheitlicher Krisen ist keine Abnahme der Nachfrage nach psychosozialer Versorgung zu erwarten. Im Gegenteil: Der Trend bewegt sich in Richtung eines steigenden Bedarfs an Behandlungsangeboten (Berufsverband Österreichischer PsychologInnen 2020). Psychotherapeutische Ausbildungs- und Universitätsambulanzen können zur Deckung der Nachfrage einen entscheidenden Beitrag leisten.

\section{Limitationen der Studie}

$\mathrm{Zu}$ den Ergebnissen ist anzumerken, dass die aktuelle Situation der Nicht-Verfügbarkeit voll kassenfinanzierter Psychotherapie in Österreich die Charakteristika der Patient*innengruppe an der SFU-Ambulanz beeinflusst: Es ist anzunehmen, dass das finanziell niederschwellige Angebot dazu führt, dass auch Patient*innen Psychotherapie in Anspruch nehmen, die bei niedergelassenen freiberuflichen Psychotherapeut*innen keine Möglichkeit der psychotherapeutischen Versorgung fänden. Inwieweit dies auf eine Vergleichbarkeit der Ergebnisse der Studie mit der Versorgungslage in anderen europäischen Ländern Einfluss nimmt und eine Generalisierbarkeit auf die Versorgungssituation in anderen Ländern daher zu hinterfragen ist, ist als Limitation anzuführen.

Interessenkonflikt M. Reisinger, F. Knasmüller, J. Aden und B. Sindelar bestätigen, dass kein Interessenkonflikt besteht.

Open Access Dieser Artikel wird unter der Creative Commons Namensnennung 4.0 International Lizenz veröffentlicht, welche die Nutzung, Vervielfältigung, Bearbeitung, Verbreitung und Wiedergabe in jeglichem Medium und Format erlaubt, sofern Sie den/die ursprünglichen Autor(en) und die Quelle ordnungsgemäß nennen, einen Link zur Creative Commons Lizenz beifügen und angeben, ob Änderungen vorgenommen wurden.

Die in diesem Artikel enthaltenen Bilder und sonstiges Drittmaterial unterliegen ebenfalls der genannten Creative Commons Lizenz, sofern sich aus der Abbildungslegende nichts anderes ergibt. Sofern das betreffende Material nicht unter der genannten Creative Commons Lizenz steht und die betreffende Handlung nicht nach gesetzlichen Vorschriften erlaubt ist, ist für die oben aufgeführten Weiterverwendungen des Materials die Einwilligung des jeweiligen Rechteinhabers einzuholen.

Weitere Details zur Lizenz entnehmen Sie bitte der Lizenzinformation auf http://creativecommons.org/licenses/by/4. $0 /$ deed.de.

\section{Literatur}

Angermeyer, M. C., Kilian, R., \& Matschinger, H. (2000). WHOQOL - 100 und WHOQOL - BREF. Handbuch für die deutschsprachige Version der WHO Instrumente zur Erfassung von Lebensqualität. Göttingen:Hogrefe.
Arnett, J. J. (2015). Emerging Adulthood. The Winding Road from the Late Teens Through the Twenties (2. Ausg.). Oxford: Oxford University Press.

Berufsverband Österreichischer PsychologInnen (2019). Zeit zu handeln: Mehr psychische Gesundheit in Österreich spart Leid und Geld. Abgerufen: 3 Nov 2020, von https://www.ots.at/presseaussendung/OTS_20191008_ OTS0092/zeit-zu-handeln-mehr-psychische-gesundheitin-oesterreich-spart-leid-und-geld-bild

Berufsverband Österreichischer PsychologInnen (2020). Psychische Gesundheit in Österreich. Abgerufen: 3 Nov 2020, von https://www.boep.or.at/download/5ef991483c15c8 588f00001a/BOEP-Studie_Psychische_Gesundheit_in_ Oesterreich.pdf

Boyce, C.J., Wood, A.M., Daly, M., \& Sedikides, C. (2015). Personality change following unemployment. Journal of Applied Psychology, 100(4), 991-1011.

Czypionka, T., Lappöhn, S., Pohl, A., \& Röhrling, G. (2016). Invaliditätspension aufgrund psychischer Erkrankungen. Wien: Institut für Höhere Studien (IHS).

Gelo, O.C., \& Manzo, S. (2015). Quantitative approaches to treatment process, change process and process-outcome research. In O. C. Gelo, A. Pritz \& B. Rieken (Hrsg.), Psychotherapy research: foundations, process, and outcome (S. 247-277). Wien/NewYork: Springer.

Glass, G.V., Smith, M.L., \& Miller, T.I. (1980). The benefits of psychotherapy. Baltimore: John Hopkins University Press.

Habimana, K., Bachner, F., Bobek, J., Ladurner, J., \& Ostermann, H. (2015). Das österreichische Gesundheitswesen im internationalen Vergleich. Wien: Bundesministerium für Gesundheit.

Hauptverband der österreichischen Sozialversicherungsträger (2011). Analyse der Versorgung psychisch Erkrankter - Projekt „Psychische Gesundheit“ - Abschlussbericht. Wien/Salzburg: Hauptverband der österreichischen Sozialversicherungsträger.

Hauptverband der österreichischen Sozialversicherungsträger (2019). Krankenstandsfälle seit 1990 nach Diagnose. Abgerufen: 20 Apr 2020, von http://www.statistik. at/web_de/statistiken/menschen_und_gesellschaft/ gesundheit/gesundheitszustand/krankenstandstage/ index.html

Hoyer, J., Velten, J., Benecke, C., Berking, M., Heinrichs, N., InAlbon, T., Margraf, J., et al. (2015). Koordination der Forschung an Hochschulambulanzen für Psychotherapie. Status quo und Agenda. Zeitschrift für Klinische Psychologie und Psychotherapie. https://doi.org/10.1026/16163443/a000308.

Klein, C., Seiss, H., Scholz, P., Gregoritsch, J., Dantendorfer, K., Wallner, E., \& Hofer, K. (2012). Strategie Psychische Gesundheit. Abgerufen: 20 Apr 2020, von https://www.sozialversicherung.at/cdscontent/load? contentid $=10008.715358 \&$ version $=1391184577$

Lambert, M. J. (Hrsg.). (2013). Bergin and Garfield's handbook of psychotherapy and behavior change. Hoboken/New Jersey: JohnWiley \& Sons.

Luborsky, L., Singer, B., \& Luborsky, L. (1975). Comparative studies of psychotherapies: Isit true that everyonehaswon and all must have prizes? Archives of general psychiatry, 32(8), 995-1008.

Onken, M., \& Ströhle, A. (2005). Komorbidität von Angst und Depression. In M. Bauer, A. Berghöfer, \& M. Adli (Hrsg.), Akute und therapieresistente Depressionen. Pharmakotherapie - Psychotherapie - Innovationen (2. Aufl., S. 105-114). Berlin, Heidelberg: Springer.

Riedel, M. (2015). Modelle der Psychotherapieversorgung in Österreich.Wien:IHS. 
Schmidt-Traub, S., \& Lex, T.-P. (2005). Angst und Depression. Kognitive Verhaltenstherapie bei Angststörungen und unipolarer Depression. Göttingen:Hogrefe.

Shapiro, D.A., \& Shapiro, D. (1982). Meta-analysis of comparative therapy outcome studies: a replication and refinement. Psychological Bulletin, 92(3), 581-604.

Smith, M.L., \& Glass, G.V. (1977). Meta-analysis of psychotherapy outcome studies. American psychologist, 32(9), 752-760.

Statistik Austria (2018). Krankenstandsfälle seit 1990 nach Diagnose. Abgerufen: 20 Apr 2020, von http://www. statistik.at/web_de/statistiken/menschen_und gesellschaft/gesundheit/gesundheitszustand/ krankenstandstage/121708.html
Wancata, J. (2017). Von Prävalenz und Versorgung psychischer Krankheiten in Österreich. https://www.meduniwien.ac. at/hp/fileadmin/sozpsychiatrie/pdf/Abschlussbericht_ WIssenschaft_Wancata.pdf.Zugegriffen:3. Juni 2020.

Wittchen, H. U., Kessler, R. C., Pfister, H., Höfler, M., \& Lieb, R. (2010). Why do people with anxiety disorders become depressed? A prospective-longitudinal community study. Acta Psychiatrica Scandinavica, 102(s406), 14-23.https:// doi.org/10.1111/j.0065-1591.2000.acp29-03.x.

Hinweis des Verlags Der Verlag bleibt in Hinblick auf geografische Zuordnungen und Gebietsbezeichnungen in veröffentlichten Karten und Institutsadressen neutral. 